\title{
Saponificación artesanal de aceites de cocina usados, provenientes del municipio de Charalá
}

\author{
Martha Yanitza Arias Rodríguez¹, Diana Marcela Ibarra-Mojica² \\ ${ }^{1}$ Ingeniera Ambiental, ${ }^{2} \mathrm{MSc}$. Ingeniería Química, Docente Escuela de Ciencias Agrícolas, \\ Pecuarias y del Medio Ambiente, Universidad Nacional Abierta y a Distancia - UNAD. \\ 1 martha_yaro@hotmail.com, ${ }^{2}$ diana.ibarra@unad.edu.co
}

\section{Resumen}

Los aceites comestibles son un ingrediente básico en la preparación diaria de alimentos; sin embargo, en pocas ocasiones se cuenta con sistemas adecuados para su manejo y disposición. Frecuentemente los aceites usados son arrojados a los desagües y cañerías, lo cual genera impactos ambientales negativos, al ser este un residuo altamente contaminante y de difícil degradación (Castrillon y Rodríguez, 2012)

El municipio de Charalá (Santander) no cuenta actualmente con una Planta de Tratamiento de Aguas Residuales, por lo que estos aceites y otros contaminantes son vertidos sin ningún tratamiento a los ríos Taquiza y Pienta. Mediante el desarrollo del presente proyecto, se evaluó la pertinencia de aprovechar el aceite usado de cocina, en la fabricación artesanal de jabón, como alternativa para promover la disminución del vertimiento de aceites usados en la población.

Inicialmente se realizaron entrevistas con propietarios y/o administradores de restaurantes, establecimientos de comidas rápidas y hogares. A partir de esto, se obtuvo una estimación de la demanda semanal de aceite y jabón; así como muestras para realizar pruebas de preparación de jabón artesanal. Como resultado, se estableció que es posible preparar jabón a partir del aceite usado de cada uno de los grupos de estudio. Adicionalmente, se evidenció que, con la producción semanal de aceite usado, sería posible elaborar jabón artesanal suficiente para suplir la demanda de restaurantes, locales de comidas rápidas y hogares, durante 1.6, 2.3 y 0,3 semanas, respectivamente.

\section{Introducción}

Inicialmente, el aceite que es vertido por los desagües pueden causar obstrucciones a los sistemas de alcantarillado, debido a la formación de una capa en las paredes internas. En los sistemas de tratamiento de aguas residuales pueden ocasionar alteraciones en los procesos y operaciones normales, causando costos de mantenimiento adicionales (Pineda y Guerrero, 2011).

Una vez en los ríos o en el mar, las grasas y aceites alteran la correlación del oxígeno y agua, ya que crea una película difícil de eliminar que impide el paso de la luz y el intercambio de oxígeno entre el aire y el agua, lo que altera los ecosistemas acuáticos poniendo en peligro múltiples especies de animales y plantas. (Díaz, 2013).

Además del valor nutricional y uso en preparación de alimentos, los aceites y grasas comestibles pueden llegar hacer materia prima para la elaboración de productos como jabones y biocombustibles (Pineda y Guerrero, 2011). El aceite vegetal y las grasas animales, por ejemplo, se perfilan como una buena opción para motores diesel, debido a su volatilidad baja y al alto número de cetano que contienen, lo que permite generar la chispa necesaria para encender estos motores ( Pineda y 
Guerrero, 2011). En la Industria del biodiesel, las grasas animales y aceites vegetales son sometidos a transesterificación (González, Gómez y Abad, 2017); sin embargo, los altos costos asociados al proceso limitan su aplicación (Ortega, Mercado y Arrieta, 2014). La preparación de jabones mediante técnicas artesanales, por otra parte, podría ser una estrategia de bajo costo para disminuir el volumen de aceite vertido en cuerpos de agua, en casos como el de municipios que no cuentan con plantas de tratamiento de aguas residuales, como lo es Charalá (Santander).

\section{Saponificación de aceites usados}

El jabón es un agente limpiador que se fabrica utilizando grasas animales y/o aceites vegetales. Ejercen una acción limpiadora de grasas en presencia del agua, gracias a que presenta componentes liposolubles e hidrosolubles (Álava, 2008).

Se usa comúnmente en productos destinados a la higiene personal y para lavar determinados objetos o tejidos. Químicamente, el jabón es la sal sódica o potásica de un ácido graso, que se obtiene por hidrólisis alcalina de los ésteres contenidos en los materiales grasos. (Guerrero, 2014).

La saponificación es una reacción química entre un ácido y una base. El jabón es obtenido de la reacción de lípido saponificable (como el aceite de cocina usado) y una base como, de la cual se producen ácidos grasos y glicerina. Los aceites vegetales son los que mejor se adaptan al proceso y según la base empleada es posible obtener jabones blandos (compuestos por sales de potasio como la Potasa, $\mathrm{KOH}$ ) o duros (compuestos por sales de sodio como la soda caustica, $\mathrm{NaOH}$ ) (López et al., 2011).

El índice de saponificación (IS) es expresado como el número de miligramos de $\mathrm{KOH}$ requeridos para saponificar los ácidos grasos libres y combinados, presentes en un gramo de grasa y ofrece una medida del peso molecular promedio de los triglicéridos que constituye la grasa. Las grasas que contienen ácidos grasos de cadena corta consumen más $\mathrm{KOH}$ en su saponificación mostrando IS más grandes y las que poseen ácidos grasos de cadena larga consumen menos álcali exhibiendo valores pequeños de Índice de saponificación. (Rodríguez, Maldonado, Muro y Miranda, 2016).

\section{Recopilación de información en campo.}

Dado que no se cuenta con información documentada de producción y vertimiento de aceites usados, inicialmente se llevó a cabo entrevistas con propietarios o administradores 5 restaurantes tradicionales en el municipio de Charalá, 5 locales de comidas rápidas y 5 viviendas. En la Figura 1 se presentan los resultados obtenidos de las entrevistas, respecto a tipo de aceite usado (a), disposición del aceite usado (b), volumen de aceite usado por semana (c) y cantidad de jabón usado por semana (d).

La mayoría de los locales de comidas rápidas y restaurantes (4 y 3 respectivamente), mostraron preferencia por el uso del aceite de la marca A; y manifestaron vender el aceite usado como insumo para actividades de ganadería (2 y 4 respectivamente). Por su parte, en los hogares se evidenció el uso preferente del aceite tipo C; siendo la disposición final predominante el vertimiento al desagüe y entrega al servicio municipal de aseo (ver Anexo 1. Registro fotográfico). 


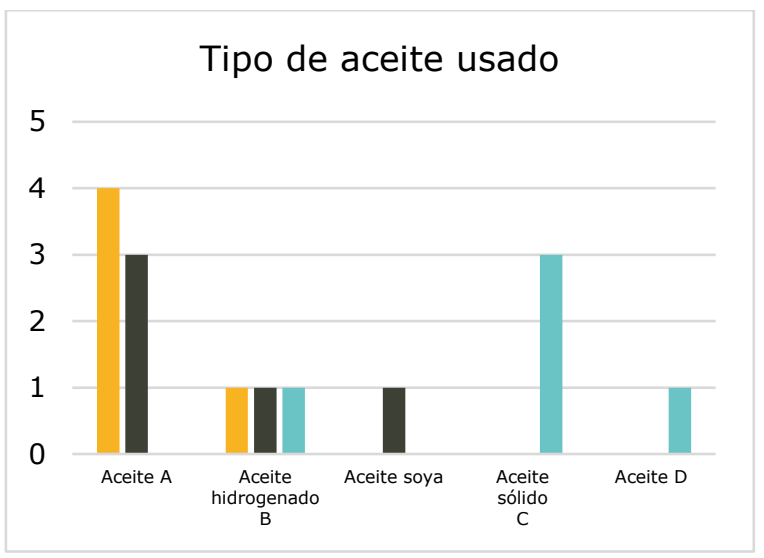

(a)

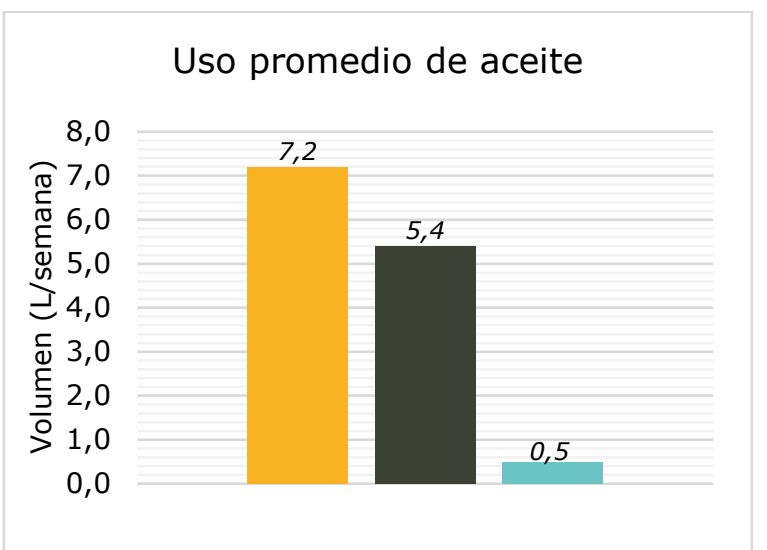

(c)

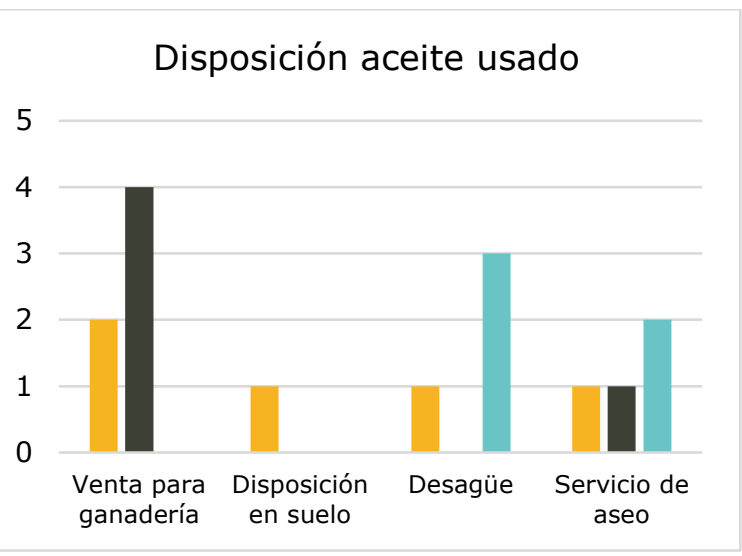

(b)

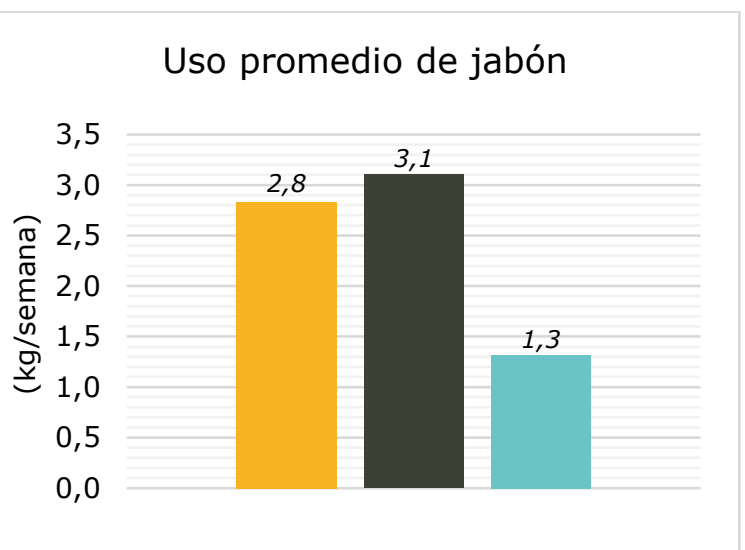

(d)

a Comidas Rápidas घRestaurantes $\quad$ Hogares

Aceite A: Mezcla de aceites de Oleína de palma y soya; Aceite B: Aceite vegetal de Soya y Oleína de Palma; Aceite C: Mezcla de aceites comestibles soya, oleína y algodón; Aceite D: Mezcla de aceites de oliva y girasol

Figura 1. Resultados de entrevistas restaurantes, locales comidas rápidas y hogares. Fuente: Autores.

La demanda de aceite fue mayor en los locales de comidas rápidas (7.2 L/semana), seguido de los restaurantes (5.4 L/semana) y finalmente los hogares $(0.5 \mathrm{~L} /$ semana); mientras que el uso de jabón fue mayor en los restaurantes $(3.1 \mathrm{~kg} / \mathrm{semana})$, seguido de los locales de comidas rápidas (2.8 $\mathrm{kg} / \mathrm{semana})$ y los hogares $(1.3 \mathrm{~kg} / \mathrm{semana})$.

La mayoría de los entrevistados manifestaron conocer impactos negativos de la inadecuada disposición final del aceite usado como: malos olores, obstrucción de las cañerías, contaminación del agua y afectación a la salud humana. Solo 3 de los entrevistados manifestaron no percibir ningún impacto negativo de esta actividad.

Finalmente, todos los entrevistados mostraron interés en aprender técnicas de saponificación artesanal del aceite usado, como una alternativa para su reutilización y reducción de demanda de jabones comerciales. 


\section{Saponificación de muestras de aceite usado, mediante técnica artesanal.}

Se recopilaron muestras de $500 \mathrm{ml}$ de aceite usado, provenientes de cada uno de los locales comerciales y hogares que aceptaron participar en las entrevistas de la fase anterior. La saponificación de las muestras se llevó a cabo mediante el "Método en frío", el cual es el más común en la fabricación de jabón artesanal dado que requiere insumos o materiales de nivel industrial (López et al., 2011) (ver Figura 2).

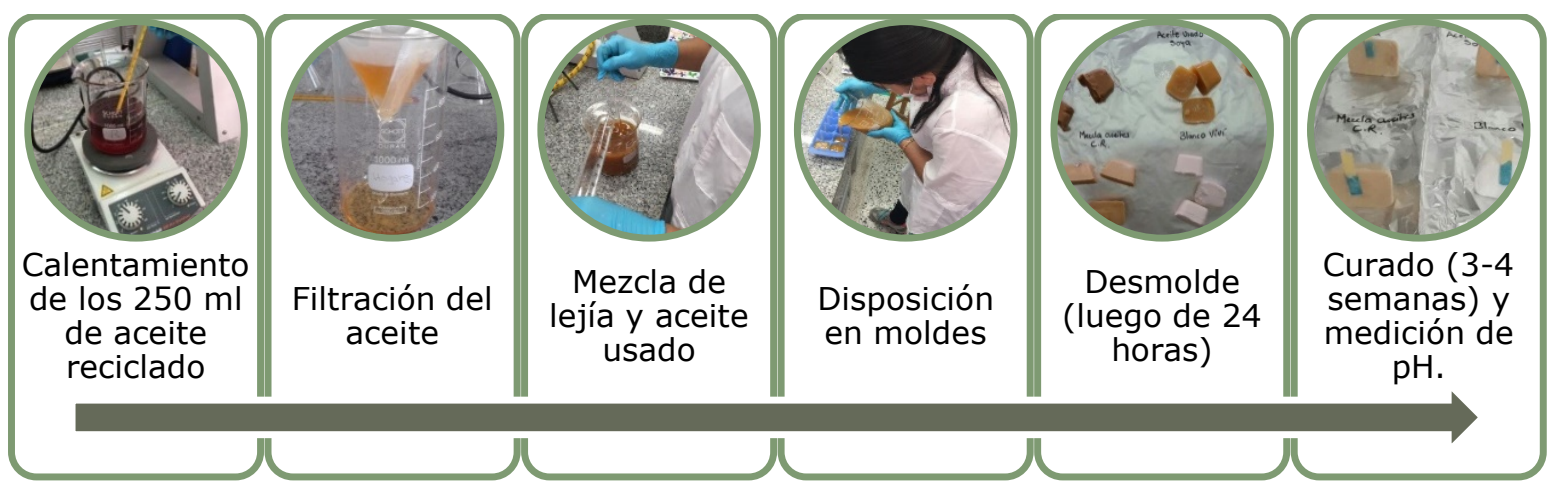

Figura 2. Saponificación con método artesanal. Fuente: Autores.

No fue posible obtener muestras usadas de cada tipo de aceite, puesto que, tanto en los hogares como en los establecimientos comerciales, los encuestados manifestaron que, a pesar de tener una preferencia por un tipo de aceite, la adquisición frecuentemente depende también del precio. Para las pruebas de saponificación se procedió a generar muestras combinadas en cada grupo de estudio y se realizaron pruebas con aceite tipo A, B, C y D no usados, a manera de comparación. En la Figura 3 se presentan las principales características observadas en los jabones obtenidos, luego de 3 semanas de secado.
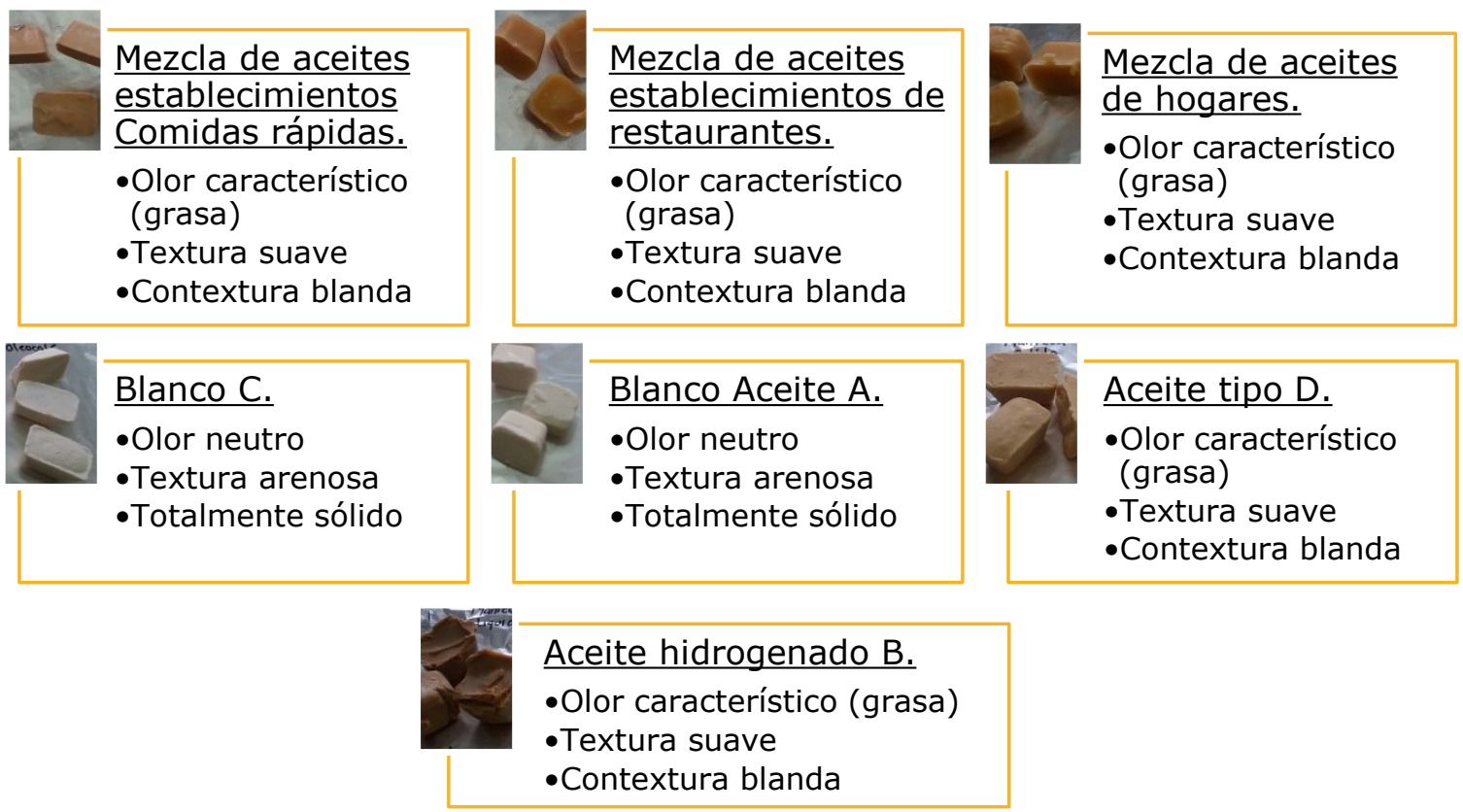

Figura 3. Resultados de saponificación artesanal. Fuente: Autores. 
Durante las 3 semanas de curado, todos los jabones mantuvieron pH neutro (7). El aceite hidrogenado $\mathrm{B}$ y aceite sólido $\mathrm{C}$ presentaron un proceso de curado más rápido (3 días); mientras que las mezclas de aceites de comidas rápidas, restaurantes y hogares aún luego de la tercera semana presentaron humedad y contextura blanda.

Finalmente, se sometieron los jabones a prueba utilizando agua. Se observó una baja generación de espuma, lo cual podría ser un factor a tener en cuenta en estudios posteriores, como una posible ventaja del uso de estos jabones para disminuir el impacto negativo en el medio ambiente.

Teniendo en cuenta que por cada $250 \mathrm{ml}$ de aceite usado se logró obtener de $225 \mathrm{gr}$ de jabón en promedio, sería posible suplir la demanda de jabón de los restaurantes, locales de comidas rápidas y hogares, durante 1.6, 2.3 y 0,3 semanas, respectivamente (ver Tabla 1).

Tabla 1. Producción de jabón artesanal Vs uso de aceite.

\begin{tabular}{lcccc}
\cline { 2 - 5 } & $\begin{array}{c}\text { Jabón usado (kg) } \\
\text { por semana }\end{array}$ & $\begin{array}{c}\text { Aceite usado } \\
\text { (L) por semana* }\end{array}$ & $\begin{array}{c}\text { Producción de } \\
\text { jabón } \mathbf{( k g )}\end{array}$ & $\begin{array}{c}\text { Semanas de } \\
\text { demanda de jabón } \\
\text { suplidas }\end{array}$ \\
\hline Restaurantes & 3,1 & 5,4 & 4,9 & 1,6 \\
Comidas Rápidas & 2,8 & 7,2 & 6,5 & 2,3 \\
Hogares & 1,3 & 0,5 & 0,5 & 0,3 \\
\hline
\end{tabular}

*Asumiendo la demanda de aceite igual a la producción de aceite usado. No se consideraron pérdidas durante la preparación de alimentos.

Fuente: Autores

\section{Conclusiones}

- Es posible preparar jabón, mediante técnicas artesanales, a partir del aceite usado de restaurantes, establecimientos de comidas rápidas y hogares, del municipio de Charalá -Santander.

- El aprovechamiento de los aceites usados en la fabricación de jabones de forma artesanal en el municipio de Charalá, podría contribuir a disminuir los vertimientos de aceite y la demanda de jabón comercial, dado que, con la producción semanal de aceite usado, sería posible suplir la demanda de jabón de los restaurantes, locales de comidas rápidas y hogares, durante 1.6, 2.3 y 0,3 semanas, respectivamente.

- Es necesario realizar trabajos adicionales para establecer la composición específica de los aceites usados y determinar los principales factores que podrían afectar la producción de jabón mediante métodos artesanales. Otros factores a evaluar serían la calidad de los jabones resultantes, los usos recomendados o restringidos y la evaluación del impacto ambiental de su uso, entre otros.

\section{Literatura citada}

Álava Ortiz, L. D. (2008). Evaluación del proceso de elaboración de jabón suave a partir de aceite rojo y residual en interacción con lejía de ceniza provenientes de la "extractora de aceite de palma africana (Elaeis guineensis jacq) QUEVEPALMA" en el canton Quevedo (Tesis de Licenicatura). Universidad Técnica Estatal de Quevedo, Quvedo, Ecuador. Recuperado de http://biblioteca.uteq.edu.ec/cgi-bin/koha/opac-detail.pl?biblionumber=1169

Castrillon Quiñones, J.A., y Rodríguez Sanz, C.A. (2012). Estimacion de la cantidad de Grasas y 
Aceites Usados de cocina y su destino en el Municipio de Pasto (Tesis de pregrado) Universidad Cooperativa de Colombia, San Juan de Pasto, Colombia.

Díaz González, A. M. (2016). Programa de gestión integral de residuos de aceite vegetal usado (AVU) y grasa animal (GA) generados en el Parque Recreativo y Zoológico Piscilago (Tesis de pregrado). Universidad de Cundinamarca, Girardot, Colombia.

González, L. V. P., Gómez, S. P. M., y Abad, P. A. G. (2017). Aprovechamiento de residuos agroindustriales en Colombia. RIAA, 8(2), 141-150.

Guerrero González, C. E. (2014). Diseño de una planta de fabricación de jabón a partir de aceites vegetales usados (Tesis de pregrado). Universidad de Almería, Almería, España. Recuperado de

http://repositorio.ual.es/bitstream/handle/10835/3371/Proyecto.pdf?sequence=1\&isAllowed=y

López, M. Z., Zamora, O. R., Pedroza, A. M., Morales, M. R., Palma, A. P., y Moreno, H. V. (2011). Elaboración de jabón en pasta de lavandería, a partir de aceite vegetal comestible de desecho, como materia prima. Naturaleza y Tecnología, 3(1), 18-25. Recuperado de http://quimica.ugto.mx/index.php/nyt/article/viewFile/38/pdf1

Ortega, G. C. C., Mercado, E. R. A., y Arrieta, B. M. C. (2014). Transesterificación de aceites vegetales empleando catalizadores heterogéneos. Prospectiva, 12(2), 90-104.

Pineda Rodriguez, C. A., y Guerrero Erazo, J. (2011). Aprovechamiento de los residuos grasos generados en los restaurantes y comidas rapidas de Pereira. Scientia et Technica, 1(47), 264269. doi: $10.22517 / 23447214.581$

Rodríguez, J. A., Maldonaado, J. M., Muro, M. A., y Miranda, L. G. 2016. Índice de saponificación de cinco mantecas determinado mediante un micrométodo. Investigación y Desarrollo en Ciencia y Tecnología de Alimentos, 1(1), 937-942. Recuperado de http://www.fcb.uanl.mx/IDCyTA/files/volume1/1/10/161.pdf 
Anexo 1. Registro fotográfico
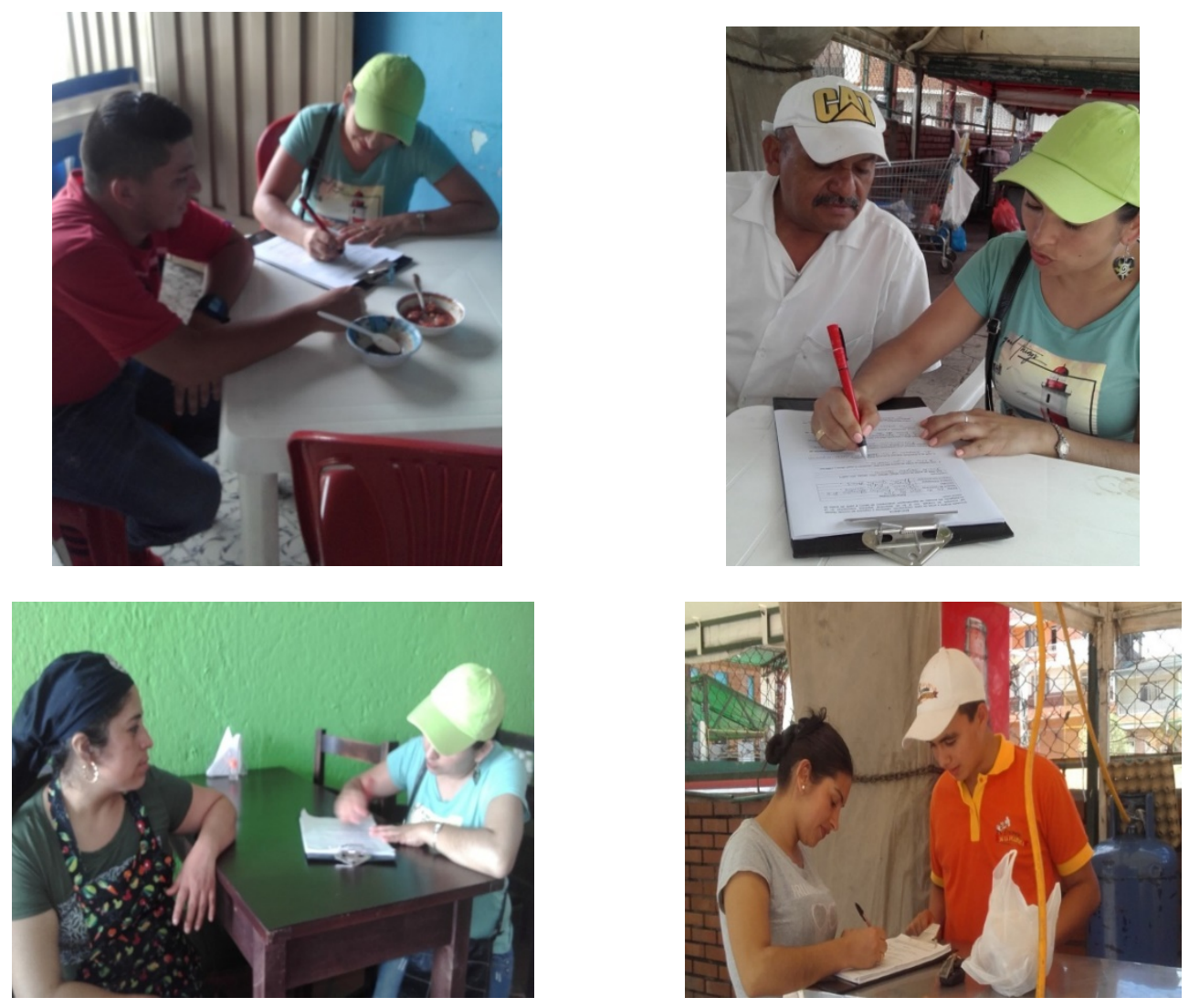